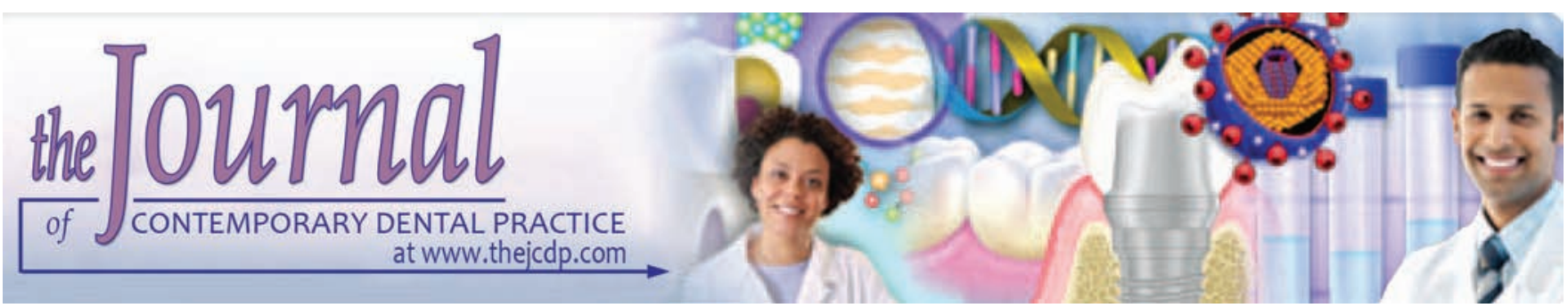

\title{
Glucose Transporter 1 Expression in Odontogenic Keratocyst, Dentigerous Cyst, and Ameloblastoma: An Immunohistochemical Study
}

\author{
${ }^{1}$ Alokenath Bandyopadhyay, ${ }^{2}$ Abikshyeet Panda, ${ }^{3}$ Shyam S Behura, ${ }^{4}$ Sujatha Ramachandra
}

${ }^{5}$ Kailash C Dash, ${ }^{6}$ Pallavi Mishra

\begin{abstract}
Introduction: An array of odontogenic lesions manifest in the maxillofacial region with variable presentations. The biological behavior of lesions, such as odontogenic keratocyst (OKC), dentigerous cyst (DC), and ameloblastoma (AM) always invite debate. Glucose transporter 1 (GLUT-1) is proven to be an indicator of metabolic behavior of several benign and malignant neoplasms.
\end{abstract}

Aim: The purpose of this study was to evaluate the expression of GLUT-1 in OKC, DC, and AM to understand their metabolic behavior.

Materials and methods: Immunohistochemical expression of GLUT-1 was evaluated in each of the 15 cases of OKC, DC, and AM. The number of labeled cells, staining intensity, and membrane or cytoplasmic expressions were the parameters assessed and analyzed using chi-square test.

Results: All cases showed positive GLUT-1 expression: 86.6\% OKC showed more than $50 \%$ labeled cells followed by DC (40\%) and AM (26.5\%); 53.3\% OKC showed strong intensity in comparison to AM, which showed weak intensity in $53.3 \%$ cases; $86.6 \%$ of OKCs showed both membrane and cytoplasmic expression followed by DC (40\%) and AM (26.6\%), whereas $73.3 \%$ of $A M$ showed only membrane expression followed by DC (60\%) and OKC (13.3\%).

Conclusion: Odontogenic keratocyst was found out to be more metabolically active followed by DC and AM.

Keywords: Ameloblastoma, Dentigerous cyst, Glucose transporter 1, Odontogenic keratocyst.

\footnotetext{
${ }^{1-6}$ Department of Oral and Maxillofacial Pathology, Kalinga Institute of Dental Sciences, Kalinga Institute of Industrial Technology University, Bhubaneswar, Odisha, India

Corresponding Author: Abikshyeet Panda, Department of Oral and Maxillofacial Pathology, Kalinga Institute of Dental Sciences, Kalinga Institute of Industrial Technology University Bhubaneswar, Odisha, India, Phone: +919040324224, e-mail: abikshyeet@yahoo.com
}

How to cite this article: Bandyopadhyay A, PandaA, Behura SS, Ramachandra S, Dash KC, Mishra P. Glucose Transporter 1 Expression in Odontogenic Keratocyst, Dentigerous Cyst, and Ameloblastoma: An Immunohistochemical Study. J Contemp Dent Pract 2017;18(5):366-370.

Source of support: Nil

Conflict of interest: None

\section{INTRODUCTION}

Pathologies originating from odontogenic tissues may vary from cyst to neoplasm, which may demonstrate distinct biological behavior. ${ }^{1}$ The most prevalent cysts encountered are radicular cyst, dentigerous cyst (DC), and odontogenic keratocyst (OKC). Whereas ameloblastomas (AMs) are the frequently reported benign odontogenic tumor, ${ }^{1}$ the OKC arises from odontogenic epithelium, which has a significant growth potential and an aggressive behavior. ${ }^{2,3} \mathrm{~A}$ DC is a developmental odontogenic cyst characterized by its slow growth and encloses the crown of an unerupted tooth at its neck. Many times they are asymptomatic. ${ }^{2,4}$ Ameloblastoma is a slow growing locally invasive benign odontogenic epithelial neoplasm with no tendency to metastasis. ${ }^{5}$ Depending on the treatment instituted, AM can have a high recurrence rate. ${ }^{1,5,6}$

Controversies have always surrounded OKC. A debate is always there to whether consider it as a cyst or a neoplasm, since it was evident that it may grow to a larger size before becoming clinically evident and has a tendency to recur following surgical treatment. Many evidences have been put forth over the years proposing OKC to be a benign cystic neoplasm. ${ }^{2}$ The World Health Organization has proposed the name keratocystic odontogenic tumor to be used in place of OKC as per the last classification proposed in $2005 .{ }^{7}$ However, this term is yet to be accepted in the literature as the molecular findings 
were not sufficiently definitive to support the lesion to be a benign neoplasm. ${ }^{2}$

Glucose is the most common primary energy molecule found on earth, which is utilized as major catabolic and anabolic substrates by a majority of organisms. ${ }^{8}$ Its entry into the cell is facilitated by a family of transporters termed as glucose transporters (GLUTs). By far, 14 members of this family have been identified. ${ }^{8,9}$ In this family, GLUT-1 is the most studied upon transporter molecule, i.e., frequently upregulated during oncogenesis of several neoplasms. ${ }^{8}$ Carcinomas of liver, pancreas, breast, brain, kidney, lungs, skin, gastrointestinal tracts, and female reproductive system show overexpression of GLUT- 1 . $^{10}$

The GLUT-1 overexpression is seen in numerous malignant as well as benign neoplasms encountered in the head and neck areas. ${ }^{11-13}$ The GLUT-1 overexpression in $\mathrm{AM}$ has also been reported using positron emission tomography scan. ${ }^{14}$ Very few studies are present in the literature enumerating the GLUT-1 expression in odontogenic cysts, such as OKC and DC. ${ }^{15,16}$ The aim of our study was to evaluate GLUT-1 expression in AM, OKC, and DC by immunohistochemistry to understand the metabolic behavior of each. This may throw some light on their biology.

\section{MATERIALS AND METHODS}

The present study was approved by the Institutional Ethics Committee, Kalinga Institute of Medical Sciences, KIIT University, Bhubaneswar, Odisha, India, Ref. No. KIMS/KIIT/IEC/49/2016. Fifteen formalin-fixed paraffin-embedded (FFPE) tissue blocks in each of DC, $\mathrm{OKC}$, and AM were obtained from the archives of the Department of Oral and Maxillofacial Pathology, Kalinga Institute of Dental Sciences. The cases had their histopathological diagnosis reviewed by two oral pathologists. Histological sections of $3 \mu \mathrm{m}$ thickness were cut from the original FFPE blocks and mounted on poly-L-lysinecoated positively charged slides before immunohistochemical staining. Antigen retrieval was performed by pressure cooker technique using Tris-ethylenediaminetetraacetic acid buffer at $\mathrm{pH} 9.0$ after deparaffinization and rehydration. The sections were then subjected to peroxide block for 15 minutes to block endogenous peroxidase activity. The GLUT-1 was immunostained using a rabbit monoclonal antibody (PathNSitu Biotechnologies Pvt. Ltd.). The incubation time was 45 minutes after which the sections were treated by PolyExcel horseradish peroxidase/diaminobenzidine detection system (PathNSitu Biotechnologies Pvt. Ltd.). Human cervical carcinoma sections and erythrocyte membranes served respectively, as external and internal positive controls.
Table 1: Parameters evaluated as adapted from Vasconcelos et al ${ }^{15}$

\begin{tabular}{ll}
\hline Parameters & Score assigned \\
\hline Percentage of & $0-$ Negative \\
labeled cells & $1-<10 \%$ \\
& $2-11-50 \%$ \\
& $3->50 \%$ \\
Intensity of staining & $1-$ Weak (+) \\
& $2-$ Moderate (++) \\
& $3-$ Strong (+++) \\
Subcellular & $1-$ Nucleus only \\
localization of & $2-$ Cytoplasm only \\
staining & $3-$ Membrane only \\
& $4-$ Combined nucleus and cytoplasm \\
& $5-$ Combined cytoplasm and membrane \\
& $6-$ Combined nucleus, cytoplasm, and \\
& membrane \\
\hline
\end{tabular}

Samples were considered positive for the expression of GLUT-1 if cells showed brown staining on the cell membrane, cytoplasm, or nucleus. Five representative areas of the epithelium were evaluated with an Olympus CX-21 microscope under 400× magnification. Following scores were used to categorize the specimen: $0(0 \%$ of positive cells), 1 ( $<10 \%$ of positive cells), 2 (11-50\% of positive cells), and 3 ( $>50 \%$ of positive cells) as per the criteria proposed by Vasconcelos et al. ${ }^{15}$ The other parameters evaluated are staining intensity and location within the positive cells. ${ }^{15}$ The parameters taken are listed in Table 1.

The results obtained were analyzed using software Statistical Package for the Social Sciences version 20.0, and statistical analysis was performed using chi-square test. A p-value $\leq 0.05$ was considered to be statistically significant.

\section{RESULTS}

After immunohistochemistry, it was observed that all the three lesions showed positive staining for GLUT-1, as shown in Figures 1 to 3 . The expression was diffuse in most of the specimens, with very few cases of AM and OKC showing focal expression pattern. All the specimens of DC showed diffuse expression pattern. In most of the OKC cases, all the cells in basal, suprabasal, intermediate, and superficial cells showed strong membrane and cytoplasmic staining, as shown in Figure 1. In AM cases, the GLUT-1 expression was confined to peripheral palisading cells and central cells (Fig. 2), whereas the intermediate area did not show expression. In DC cases, the positive cells were confined to basal area, few cells in suprabasal areas showed positive expression (Fig. 3). The expression pattern was quantified and the results obtained were tabulated in Table 2.

When percentages of labeled cells were evaluated, $86.6 \%$ OKC showed more than $50 \%$ labeled cells, 


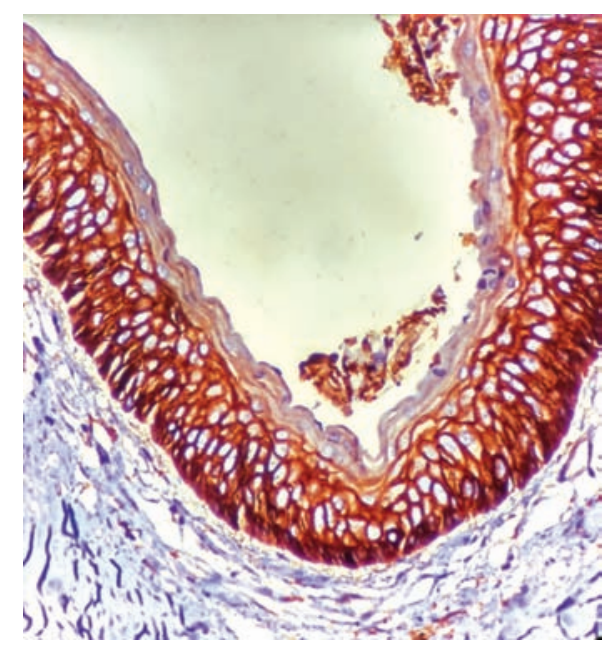

Fig. 1: Glucose transporter 1 expression in OKC (400× magnification)

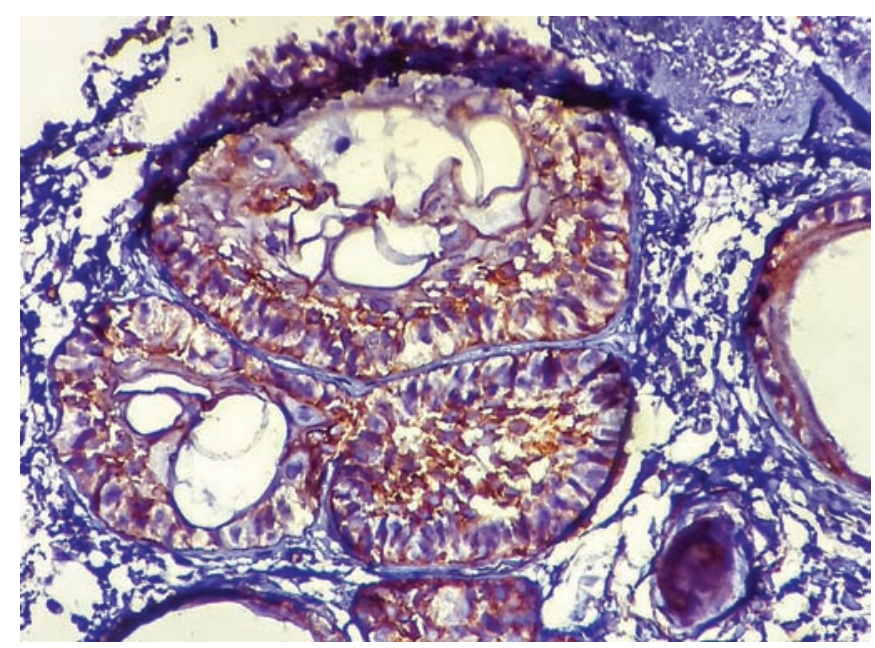

Fig. 3: Glucose transporter 1 expression in AM (400× magnification)

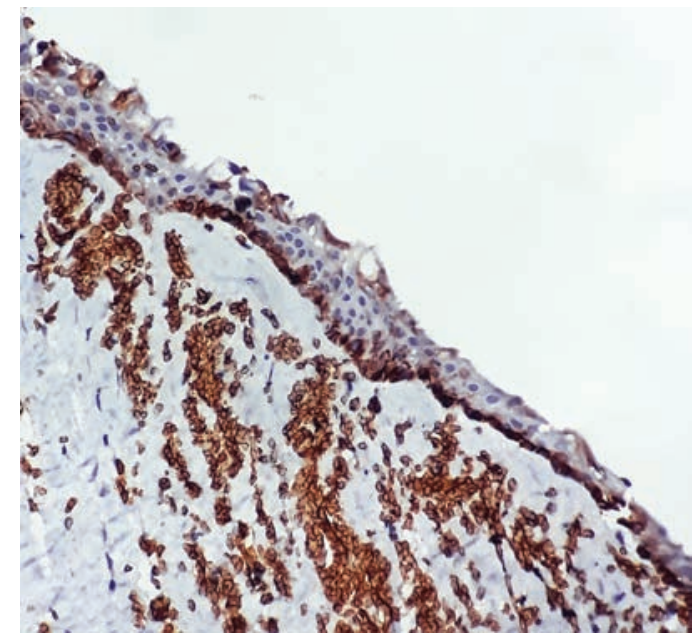

Fig. 2: Glucose transporter 1 expression in DC (400× magnification)

followed by DC (40\%) and AM (26.5\%), whereas 73.3\% AM showed 11 to $50 \%$ labeled cells. There is a statistically significant difference noticed between the groups with a $\mathrm{p}$-value of 0.003 . When staining intensity was evaluated, $53.3 \%$ OKC showed strong intensity in comparison to AM, which showed weak intensity in $53.3 \%$ cases; $40 \%$ cases of DC showed strong intensity and $60 \%$ cases showed weak intensity. When the groups were compared, a p-value of 0.011 was obtained, which showed a statistically significant difference. Most of the cases of OKC, DC, and $\mathrm{AM}$ showed diffuse expression pattern in contrast to focal. There is no statistically significant difference noticed across the groups $(p=0.099)$. On the evaluation of subcellular localization, $86.6 \%$ of OKCs showed both membrane and cytoplasmic expression followed by DC

Table 2: GLUT-1 expression pattern in OKC, DC, and AM percentage of labeled cells

\begin{tabular}{llllll}
\hline Type of lesion & Absent & $<10 \%$ & $11-50 \%$ & $>50 \%$ & $p$-value \\
\hline Odontogenic keratocyst & 0 & 0 & $2(13.3 \%)$ & $13(86.6 \%)$ & 0.003 \\
Dentigerous cyst & 0 & 0 & $9(60 \%)$ & $6(40 \%)$ & $4(26.6 \%)$
\end{tabular}

Intensity of scoring

\begin{tabular}{|c|c|c|c|c|}
\hline Type of lesion & Weak (+) & Moderate $(++)$ & Strong $(+++)$ & $p$-value \\
\hline Odontogenic keratocyst & $2(13.3 \%)$ & $5(33.3 \%)$ & $8(53.3 \%)$ & 0.011 \\
\hline Dentigerous cyst & $9(60 \%)$ & $0(0 \%)$ & $6(40 \%)$ & \\
\hline Ameloblastoma & $8(53.3)$ & $5(33.3 \%)$ & $2(13.3 \%)$ & \\
\hline
\end{tabular}

Expression pattern

\begin{tabular}{llll}
\hline Type of lesion & Diffuse & Focal & $p$-value \\
\hline Odontogenic keratocyst & $13(86.6 \%)$ & $2(13.3 \%)$ & 0.099 \\
Dentigerous cyst & $15(100 \%)$ & $0(0 \%)$ & $4(26.6 \%)$
\end{tabular}

Subcellular localization

\begin{tabular}{llll}
\hline Type of lesion & Membrane & Membrane and cytoplasm & $p$-value \\
\hline Odontogenic keratocyst & $2(13.3 \%)$ & $13(86.6 \%)$ & 0.003 \\
Dentigerous cyst & $9(60 \%)$ & $6(40 \%)$ & \\
Ameloblastoma & $11(73.3 \%)$ & $4(26.6 \%)$ & \\
\hline
\end{tabular}


(40\%) and AM (26.6\%), whereas $73.3 \%$ of AM showed only membrane expression followed by DC (60\%) and OKC (13.3\%). The difference in subcellular localization was found to be statistically significant across the groups with a p-value of 0.003 .

\section{DISCUSSION}

Odontogenic keratocyst has a distinct aggressive behavior and shows a marked proliferative activity. ${ }^{17-20}$ The high infiltrative growth of OKC was comparable to AM. Due to this nature of $\mathrm{OKC}$, a change in its terminology was long proposed and suggestions were made to consider it to be a neoplasm instead of a cyst. ${ }^{7}$

A neoplastic cell has numerous properties, which make it self-sustaining. High metabolic activity can be one of them. Malignant cells require energy to proliferate and survive which is generated from glucose. ${ }^{10}$ This high demand of glucose is met by transport of glucose into the neoplastic cells by a specific group of transport molecules termed as GLUTs. ${ }^{8}$ In the cells, GLUTs are stored in specialized endosomes and when stimulated become an integral protein plasma membrane to start glucose intake. They are under the regulation of hormones as well as metabolic signals. ${ }^{21,22}$ In this family, GLUT- 1 is the most exhaustively studied transport molecule, which is overexpressed in neoplastic cells. ${ }^{9}$ Malignant cells show a high expression of GLUT-1 molecules, which explains their increased glucose consumption to provide energy for cell proliferation and tumor growth. ${ }^{23-26}$

Even a few benign neoplasms of the head and neck area, such as pleomorphic adenoma ${ }^{11}$ and ceruminous adenoma, ${ }^{12}$ also express GLUT-1. Odontogenic neoplasms, such as calcifying cystic odontogenic tumor also showed GLUT-1 expression. ${ }^{27}$ Otsuru et $\mathrm{al}^{14}$ found out mild to moderate 18F-fluorodeoxyglucose (FDG) uptake in AM, correlating it with GLUT-1 expression. Seno et $\mathrm{al}^{28}$ also reported a mild FDG uptake in case of AM, correlating the benign nature of this locally invasive tumor. Sánchez-Romero et $\mathrm{al}^{29}$ found out both membrane and cytoplasmic positivity of GLUT-1 in AM.

The GLUT-1 expression is also seen in erythrocytes, endothelium, blood-brain barrier, basal layer of the oral epithelium and epidermis, and tooth germs. ${ }^{29-32}$ This indicates that even normal cells with high metabolic activities express GLUT-1. Hence, GLUT-1 can be considered as a marker of high metabolic activity as well.

Moreover, GLUT-1 facilitates increased glucose transport when induced by conditions, such as cell division, differentiation, nutrient starvation, hypoxia, and malignant transformation. Upon induction, there will be a receptor unmasking at cell membrane level. An increase in demand leads to translocation of GLUT-1 from cytoplasm to cell membrane. This leads to more GLUT-1 messenger ribonucleic acid synthesis and increased cytoplasmic GLUT-1 concentration to meet the demand. The result is increased GLUT-1 expression, in both cytoplasm and cell membrane. ${ }^{29}$

In the present study, when the GLUT-1 expression was evaluated in $\mathrm{OKC}$, it showed more number of labeled cells, strong intensity of staining, and both membrane and cytoplasmic staining. Similar findings were also reported by Vasconcelos et $\mathrm{al}^{15}$ and Vera-Sirera et al. ${ }^{16}$ When the number of labeled cells was considered, in contrast to OKC, AM and DC showed less number of labeled cells. However, Vasconcelos et $\mathrm{al}^{15}$ found all three lesions expressing more number of labeled cells. Vera-Sirera et $\mathrm{al}^{16}$ compared the GLUT-1 expression of OKC with orthokeratinized odontogenic cyst and found higher expression in OKC. They proposed that the higher expression of GLUT-1 in OKC can be attributed to the high cellular proliferation as seen in various other types of tumors.

In their study of GLUT-1 expression in AM, SánchezRomero et $\mathrm{al}^{29}$ found positive membrane and cytoplasmic expression of GLUT-1 in all the cases of AM with moderate to intense staining. However, in our study, AM showed less number of labeled cells with weak staining intensity and mostly membrane expression. This was in contrast to the findings given by Sánchez-Romero et al. ${ }^{29}$

In our study, it was quite evident that OKC shows a higher expression of GLUT-1. Both membrane and cytoplasmic expression were noticed in all the layers of the cystic lining. Definitely the epithelial component has high demand for energy so as to perform its high proliferative activity. This explains the invasiveness and aggressive nature of $\mathrm{OKC}$. In that way, the biological behavior of OKC can be comparable to various other benign and malignant neoplasms as outlined by various authors. ${ }^{22-}$ ${ }^{26}$ Hence, increased GLUT-1 expression in OKC may be treated as molecular evidence to justify the neoplastic nature of OKC, as demanded by various authors in $2007 .^{2}$

On the contrary, the decreased GLUT-1 expression in $\mathrm{AM}$ is inviting further debate in this very same context. A larger study with more number of samples may clear the picture further.

\section{CONCLUSION}

Increased GLUT-1 expression is seen in OKC followed by DC. In our study, AM showed less expression of GLUT-1. In a neoplastic environment, rapid proliferation may lead to hypoxia, which results in increased expression of GLUT-1 in both cytoplasm and membrane. There is an increased membrane and cytoplasm expression seen in OKC in comparison to DC and AM. Thus, OKC may be considered as more aggressive with high metabolic activity, which may explain its infiltrative behavior and higher rate of recurrence. 


\section{REFERENCES}

1. Johnson NR, Gannon OM, Savage NW, Batstone MD. Frequency of odontogenic cysts and tumors: a systematic review. J Investig Clin Dent 2014 Feb;5(1):9-14.

2. Shear, M.; Speight, P. Odontogenic keratocyst. In: Cysts of the oral and maxillofacial regions. 4th ed. Singapore: Blackwell Munksgaard; 2007. p. 6-58.

3. Sánchez-BurgosR,González-Martín-MoroJ,Pérez-FernándezE, Burgueño-García M. Clinical, radiological and therapeutic features of keratocystic odontogenic tumours: a study over a decade. J Clin Exp Dent 2014 Jul;6(3):e259-e264.

4. Selvamani M, Donoghue M, Basandi PS. Analysis of 153 cases of odontogenic cysts in a South Indian sample population: a retrospective study over a decade. Braz Oral Res 2012 Jun;26(4):330-334.

5. Reichart, PA.; Philipsen, HP. Solid multicystic ameloblastoma. In: Odontogenic tumors and allied lesions. 1st ed. London: Quintessence Publishing; 2004. p. 43-58.

6. de Santana Santos T, Piva MR, de Souza Andrade ES, Vajgel A, Vasconcelos RJ, Martins-Filho PR. Ameloblastoma in the northeast region of Brazil: a review of 112 cases. J Oral Maxillofac Pathol 2014 Sep;18(Suppl 1):S66-S71.

7. Barnes, L.; Eveson, JW.; Reichart, P.; Sidransky, D. World Health Organization classification of tumours. Pathology and genetics of head and neck tumours. Lyon: IARC; 2005.

8. Thorens B, Mueckler M. Glucose transporters in the 21stcentury. Am J Physiol Endocrinol Metab 2010 Feb;298(2):E141-E145.

9. Wood IS, Trayhurn P. Glucose transporters (GLUT and SGLT): expanded families of sugar transport proteins. Br J Nutr 2003 Jan;89(1):3-9.

10. Carvalho KC, Cunha IW, Rocha RM, Ayala FR, Cajaíba MM, Begnami MD, Vilela RS, Paiva GR, Andrade RG, Soares FA. GLUT1 expression in malignant tumors and its use as an immunodiagnostic marker. Clinics (Sao Paulo) 2011 Jun;66(6): 965-972.

11. Horiuchi C, Tsukuda M, Taguchi T, Ishiguro Y, Okudera $K$, Inoue T. Correlation between FDG-PET findings and GLUT1 expression in salivary gland pleomorphic adenomas. Ann Nucl Med 2008 Oct;22(8):693-698.

12. Shen WQ, Cheng KJ, Bao YY, Zhou SH, Yao HT. Expression of Glut-1, HIF-1a, PI3K and p-Akt in a case of ceruminous adenoma. Head Neck Oncol 2012 May;4(1):18.

13. Azad N, Kumari Maurya M, Kar M, Goel MM, Singh AK, Sagar M, Mehrotra D, Kumar V. Expression of GLUT-1 in oral squamous cell carcinoma in tobacco and non-tobacco users. J Oral Biol Craniofac Res 2016 Jan-Apr;6(1):24-30.

14. Otsuru M, Aoki T, Tsukinoki K, Ota Y, Karakida K, Yamazaki H, Yasuda M, Kaneko A. Usefulness of 18F-fluorodeoxyglucose positron emission tomography for detecting ameloblastoma, with special reference to glucose transporter-1 expression. J Oral Maxillofac Surg 2008 Aug;66(8):1761-1765.

15. Vasconcelos RC, de Oliveira Moura JM, Lacerda Brasileiro Junior V, da Silveira ÉJ, de Souza LB. Immunohistochemical expression of GLUT-1, GLUT-3, and carbonic anhydrase IX in benign odontogenic lesions. J Oral Pathol Med 2016 Oct;45(9):712-717.

16. Vera-Sirera B, Forner-Navarro L, Vera-Sempere F. Immunohistochemical expression of glucose transporter 1 in keratin-producing odontogenic cysts. BMC Oral Health 2016 Dec;16(1):32.

17. Swetha P, Ramesh K, Madhavan N, Veeravarmal V, Sameera A. Expression of inducible nitric oxide synthase in the epithelial linings of odontogenic keratocyst, dentigerous cyst and radicular cyst: a pathological insight. Ann Med Health Sci Res 2014 Jul-Aug;4(4):583-589.

18. Shear M. The aggressive nature of the odontogenic keratocyst: is it a benign cystic neoplasm? Part 1: clinical and early experimental evidence of aggressive behaviour. Oral Oncol 2002 Apr;38(3):219-226.

19. Gani F, Mahomed F, Meer S. Evaluation of Ki-67 and cyclin D1 expression in odontogenic keratocysts and orthokeratinised jaw cysts. SADJ 2012 Aug;67(7):370-373.

20. Vera-Sirera B, Forner-Navarro L, Vera-Sempere F. Differential expression of cyclin D1 in keratin-producing odontogenic cysts. Med Oral Patol Oral Cir Bucal 2015 Jan;20(1):e59-e65.

21. Zhao FQ, Keating AF. Functional properties and genomics of glucose transporters. Curr Genomics 2007 Apr;8(2):113-128.

22. Eckert AW, Lautner MHW, Schütze A, Taubert H, Schubert J, Bilkenroth U. Coexpression of hypoxia-inducible factor-1a and glucose transporter-1 is associated with poor prognosis in oral squamous cell carcinoma patients. Histopathology 2011 Mar;58(7):1136-1147.

23. Smith TA. Facilitative glucose transporter expression in human cancer tissue. Br J Biomed Sci 1999 Feb;56(4):285-292.

24. Ayala FR, Rocha RM, Carvalho KC, Carvalho AL, da Cunha IW, Lourenço SV, Soares FA. GLUT1 and GLUT3 as potential prognostic markers for oral squamous cell carcinoma. Molecules 2010 Apr;15(4):2374-2387.

25. Salla JT, Johann AC, Lana AM, do Carmo MA, Nunes FD, Mesquita RA. Immunohistochemical study of GLUT-1 in oral peripheral nerve sheath tumors. Oral Dis 2008 Sep;14(6): 510-513.

26. Parente P, Coli A, Massi G, Mangoni A, Fabrizi MM, Bigotti G. Immunohistochemical expression of the glucose transporters GLUT-1 and GLUT-3 in human malignant melanomas and benign melanocytic lesions. J Exp Clin Cancer Res 2008 Sep;27(1):34.

27. RumayorA,Carlos R,KirschHM, deAndradeBA, RomañachMJ, de Almeida OP. Ghost cells in pilomatrixoma, craniopharyngioma, and calcifying cystic odontogenic tumor: histological, immunohistochemical, and ultrastructural study. J Oral Pathol Med 2015 Apr;44(4):284-290.

28. Seno S, Kitajima K, Inokuchi G, Nibu K, Itoh T, Ejima Y, Sasaki R, Sugimoto K, Sugimura K. FDG-PET findings of ameloblastoma: a case report. Springerplus 2015 Jun;4:250.

29. Sánchez-Romero C, Bologna-Molina R, Mosqueda-Taylor A, Paes de Almeida O. Immunohistochemical expression of GLUT-1 and HIF-1a in tooth germ, ameloblastoma, and ameloblastic carcinoma. Int J Surg Pathol 2016 Aug;24(5):410-418.

30. Abdou AG, Eldien MM, Elsakka D. GLUT-1 expression in cutaneous basal and squamous cell carcinomas. Int J Surg Pathol 2015 Sep;23(6):447-453.

31. Angadi VC, Angadi PV. GLUT-1 immunoexpression in oral epithelial dysplasia, oral squamous cell carcinoma, and verrucous carcinoma. J Oral Sci 2015 Jun;57(2):115-122.

32. Piña AR, Martínez MM, de Almeida OP. Glut-1, best immunohistochemical marker for perineurial cells. Head Neck Pathol 2015 Mar;9(1):104-106. 